\title{
Evaluating Usefulness of 5-Aminolevulinic Acid induced Fluorescence to guide Biopsy of Oral Cancers and Premalignant Lesions
}

\author{
${ }^{1}$ Manaswini Ramachandra, ${ }^{2} \mathrm{SM}$ Azeem Mohiyuddin, ${ }^{3} \mathrm{TN}$ Suresh, ${ }^{4} \mathrm{~A}$ Sagayaraj, ${ }^{5}$ Shuaib Merchant
}

\begin{abstract}
Background: Oral carcinoma prevalence is high in Kolar owing to tobacco chewing habit. The patients may have premalignant lesions all over oral cavity (field cancerization) making it difficult to select ideal biopsy site. 5-aminolevulinic acid (5-ALA) induced fluorescence helps to demarcate lesions beyond visible margin and helps to choose biopsy site.
\end{abstract}

Objectives: To perform separate biopsies from the visible and fluorescent margins of malignant/premalignant lesions of oral cavity following staining with 5-ALA and determine if microscopic tumor extends beyond visible margin.

Materials and methods: Our study included 50 patients with oral carcinoma/ premalignant lesions. Biopsies were taken from visible and fluorescence margins following incubation period of 3 hours post rinsing with 5-ALA solution. Specimens subjected to histopathological examination for detection of malignant cells. Usefulness of 5-ALA was documented by evaluating sensitivity, specificity, positive and negative predictive values and diagnostic accuracy.

Results: Fifty patients with age ranging from 33 to 78 years showed female predominance-4:1. Forty-seven patients noted fluorescence beyond visible margin. Tongue malignancies showed maximum extension. Diagnostic sensitivity with 5-ALA was $95.74 \%$ and specificity was $100 \%$. Positive and negative predictive values were 100 and $60 \%$ respectively. 5-ALA's diagnostic accuracy was determined to be $96 \%$.

Conclusion: Majority patients showed microscopic tumor extension beyond visible margin. 5-ALA staining helps in determining the true extent of lesion as Protoporphyrin IX accumulation in malignant cells shows fluorescence. This helps in guiding biopsies in field cancerization and adequate surgical tumor clearance thereby improving locoregional control.

Keywords: 5-aminolevulinic acid, Biopsy, Fluorescence, Oral carcinoma, Protoporphyrin IX, Premalignant lesion.

How to cite this article: Ramachandra M, Mohiyuddin SMA, Suresh TN, Sagayaraj A, Merchant S. Evaluating Usefulness of

\footnotetext{
${ }^{1}$ Postgraduate Student, ${ }^{2,3}$ Professor, ${ }^{4,5}$ Associate Professor

1,2,4,5 Department of Otorhinolaryngology, Sri Devaraj Urs Medical College, Kolar, Karnataka, India

${ }^{3}$ Department of Pathology, Sri Devaraj Urs Medical College Kolar, Karnataka, India

Corresponding Author: SM Azeem Mohiyuddin, Professor Department of Otorhinolaryngology, Sri Devaraj Urs Medical College, Kolar, Karnataka, India, Phone: 9845373279 , e-mail: azeem_hn@yahoo.co.in
}

5-Aminolevulinic Acid induced Fluorescence to guide Biopsy of Oral Cancers and Premalignant Lesions. Int J Head Neck Surg 2015;6(2):64-68.

\section{Source of support: Nil}

Conflict of interest: None

\section{INTRODUCTION}

Oral cancer is the most common cancer in males and 3rd most common in females in India. Squamous cell carcinoma (SCC) is the most common type (>90\%) with a male: female ratio of 2:1. It is generally seen in people above 40 years and peaking at around 60 years of age. There is very high prevalence of oral cancer in Kolar due to the habit of tobacco chewing. Alcohol and tobacco play an important synergistic role in the pathogenesis.

A large number of patients presenting to our institution have associated premalignant lesions in oral cavity. In patients with premalignant lesions over a large area, it is often difficult to choose a site for biopsy. Biopsy can be made more representative by using supravital stainslugol's iodine, toluidine blue, methylene blue, etc. Of late, hematoporphyrin derivatives and photosensitizers like 5-aminolevulinic acid (5-ALA) have been used for staining as it induces fluorescence in malignant lesions due to preferential protoporphyrin (PP) accumulation. This helps in selecting the site for biopsy, demarcation of tumor margin and in follow-up after treatment. It also facilitates early detection of malignancies and may ensure adequate clearance during surgery. 5-aminolevulinic acid hydrochloride is a naturally occurring substance and can be applied locally. Even on systemic administration it gets metabolized and cleared within 24 hours. Literature shows that 5-ALA induced fluorescence has high specificity for neoplastic tissue and no major adverse effects.

We wanted to study the usefulness of 5-ALA induced fluorescence in demarcating the tumor margins in oral cancer and find out whether microscopic tumor extended beyond the visible margins.

\section{MATERIALS AND METHODS}

This observational study was performed on 50 patients with malignancy/premalignant lesions of oral cavity at RL Jalappa Hospital and Research Centre, Kolar, India, 
between November 2011 and March 2013. It included 30 patients with oral cancer and 20 patients with premalignant lesions. Female predominance was noted (80\%). Patients with recurrent disease or earlier treatment like chemotherapy/radiotherapy and nonsquamous malignancies were excluded from the study.

An informed written consent was obtained from the patients included in the study.

Patients were made to rinse their oral cavity on the side of the lesion with 5-ALA solution (200 mg in $50 \mathrm{ml}$ of water) for 15 minutes. After incubation period of 3 hours, biopsies were taken from margin of visible lesion in patients with cancer and/or precancerous lesions of oral cavity (Fig. 1) and another from margin of 5-ALA induced fluorescence area under guidance of blue excitation light (Fig. 2). The distance in millimeters between visible and fluorescent margin was documented and the biopsy specimens were sent for histopathological examination for detection of malignant or premalignant cells in both the specimens. The results were documented. Usefulness of 5-ALA was evaluated by calculating the sensitivity, specificity, positive predictive value, negative predictive value and diagnostic accuracy.

\section{RESULTS}

This study, done at RL Jalappa Hospital, Kolar, India, included a total number of 50 patients (30 oral malignancies and 20 premalignant conditions of oral cavity). Eighty percent of patients were females and majority were in 4th to 6th decades of life. Buccal mucosa was the site of predilection.

Out of the 30 patients with malignancy, 23 were squamous carcinoma of buccal mucosa, three of malignancy tongue, one of malignancy hard palate, two of lower alveolus malignancy and one of malignancy retromolar trigone. Among the 20 patients with premalignant

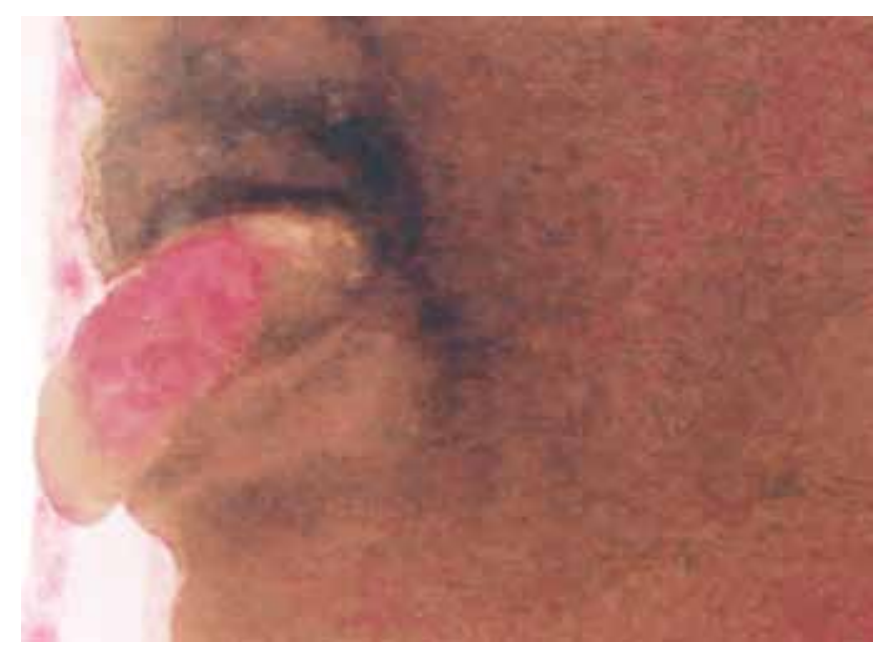

Fig. 1: Clinical examination of malignancy tongue under white light lesions, leukoplakia buccal mucosa constituted 11 cases and leukoplakia tongue was noted in one case. Six cases were of erythroplakia buccal mucosa and two of erythroleukoplakia buccal mucosa. Carcinoma oral cavity is the most common malignancy in Kolar district.

Malignancy buccal mucosa was observed to be the most afflicted subsite (23/30 malignancy patients). Patients in this region usually present at an advanced stageT3N0: two patients, T3N1: six patients, T3N2a and T3N2b: one patient each; T4aN0: two patients, T4aN1: six patients, T4aN2a: one patient. The tumor node metastasis (TNM) staging of malignancy tongue consisting of three patients was T2N0, T3N1 and T4aN1. This late presentation is due to poverty and ignorance of the people of this region.

Forty-seven out of 50 patients were found to have extension (fluorescence) beyond visible margin when visualized with the aid of blue wavelength light (Table 1). Majority of the cases-31 (66\%) showed extension between 3 and $5 \mathrm{~mm}$ beyond visible margin. All cases of malignancy (30 patients) showed extension (fluorescence) beyond visible margin. Histopathology of biopsy taken from fluorescent margin showed frank malignancy in 18 patients and dysplasia in 12 patients. Sixteen cases of premalignancy buccal mucosa and two case of premalignancy tongue demonstrated fluorescence beyond visible margin. Histopathology report of two premalignancy (leukoplakia) left buccal mucosa showed no evidence of dysplasia on biopsy from visible margin.

In our series, three patients had no fluorescence beyond visible margin and in two patients the biopsy taken from fluorescent margin did not show any dysplasia. All these five patients were clinically diagnosed as leukoplakia buccal mucosa. Twenty-seven cases (15premalignancy and 12-malignancy) demonstrated dysplasia on histopathological examination of biopsy from fluorescent margin (extension beyond visible margin).

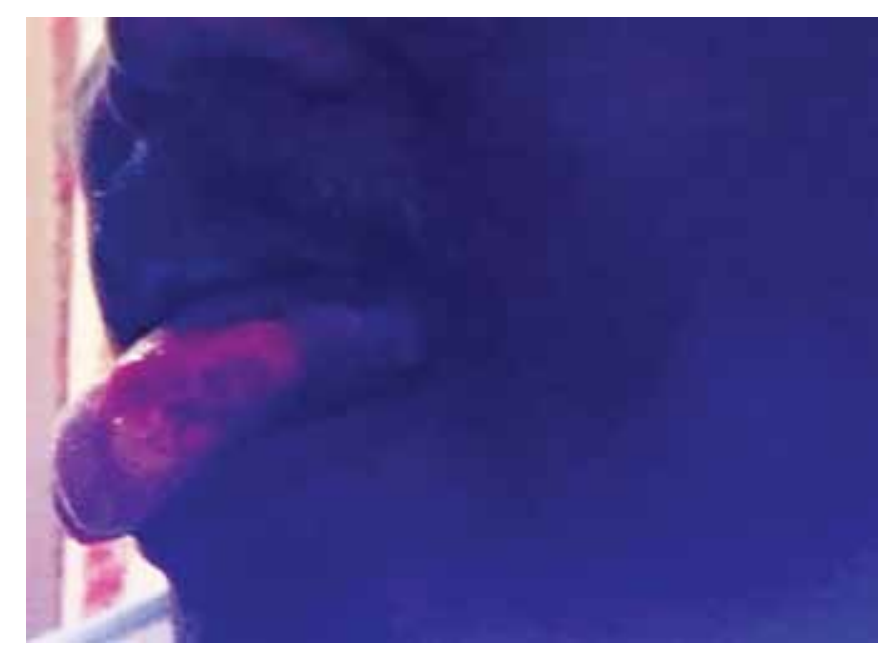

Fig. 2: Examination of malignancy tongue under blue wavelength light 
Table 1: Depiction of histopathology reports of biopsy from fluorescence margin $(n=50)$

\begin{tabular}{lll}
\hline $\begin{array}{l}\text { Results of biopsy from fluorescence } \\
\text { margin }\end{array}$ & $\begin{array}{l}\text { No. of } \\
\text { cases }\end{array}$ & $\begin{array}{l}\text { Fluorescence/ } \\
\text { visible margin }\end{array}$ \\
\hline Well differentiated SCC [A] & 8 & $8 / 14$ \\
Well to moderately differentiated & 6 & $6 / 9$ \\
SCC [B] & & \\
Moderately differentiated SCC [C] & 2 & $2 / 3$ \\
Poorly differentiated SCC [D] & 1 & $1 / 1$ \\
Verrucous carcinoma [E] & 1 & $1 / 1$ \\
Severe dysplasia [F] & 7 & $7 / 5$ \\
Moderate to severe dysplasia [G] & 2 & $2 / 1$ \\
Moderate dysplasia [H] & 6 & $6 / 5$ \\
Mild to moderate dysplasia [I] & 3 & $3 / 4$ \\
Mild dysplasia [J] & 9 & $9 / 4$ \\
No evidence of dysplasia [K] & 2 & $2 / 3$ \\
No extension [L] & 3 & - \\
\hline
\end{tabular}

Table 2: Subsites showing $>5 \mathrm{~mm}$ extension from visible margin $(n=8)$

\begin{tabular}{lll}
\hline Subsites & $\begin{array}{l}\text { No. of } \\
\text { cases }\end{array}$ & $\begin{array}{l}\text { Average distance of extension } \\
\text { (by fluorescence) in } \mathrm{mm}\end{array}$ \\
\hline $\begin{array}{l}\text { Malignancy buccal } \\
\text { mucosa }\end{array}$ & 5 & $6.6 \mathrm{~mm}(6-7 \mathrm{~mm})$ \\
Malignancy tongue & 3 & $11 \mathrm{~mm}(8-13 \mathrm{~mm})$ \\
\hline
\end{tabular}

Eight patients in our study had $>5 \mathrm{~mm}$ extension (fluorescence) beyond visible margin. The subsites showing $>5 \mathrm{~mm}$ extension of fluorescence constituted five cases of malignancy buccal mucosa and three cases of malignancy tongue (Table 2). It is to be noted here that all three cases of malignancy tongue that were taken in this study showed an extension of $>5 \mathrm{~mm}$ from visible margin on fluorescence staining.

In our study, in biopsies taken from areas of field cancerization in three cases, two cases of leukoplakia showed dysplasia and one case of erythroplakia showed severe dysplasia. In these three patients, the appropriate site for biopsy could be selected only after staining with 5-ALA.

\section{DISCUSSION}

Supravital dyes-toluidine blue, Lugol's iodine, methylene blue, ALA and other porphyrins-have been used to determine the presence of malignancy and its extension beyond the gross margin. ${ }^{1}$ They serve as a means of surveillance for high-risk cases and also for those diagnosed with neoplasm of other parts of the aerodigestive tract. ${ }^{2}$

Toluidine blue is taken up by dysplastic cells owing to their DNA binding property. ${ }^{3}$ Studies done show sensitivity varying between 91 to 92 and $100 \%$ specificity. Diagnostic accuracy of $65 \%$ was observed. ${ }^{4,5}$ In 1989 , a meta-analysis wherein the sensitivity was $97.7 \pm 4.65 \%$ and specificity was $90.8 \pm 9.34 \% .{ }^{6}$ False positive results occur due to inflammatory and ulcerative lesions which retain the dye. ${ }^{6}$
Lugol's iodine was the earliest dye to be used for staining tissues for detection of dysplastic/malignant cells. This dye does not cause staining of malignant/ dysplastic cells due to the enhanced glycolysis occurring in these cells. Its usefulness is confined to nonkeratinized mucosa. ${ }^{1}$ The pioneer study in 1990, wherein 178 patients were screened by lugol's iodine staining, identified 13 cases with oral cancer and dysplasia. ${ }^{1}$

A study comparing Lugol's iodine and toludine blue done at University of British Columbia, Vancouver, which included 59 cases, concluded that lugol's iodine was less sensitive but had greater specificity.

Action of methylene blue is similar to toluidine blue and is preferred for large scale screening. ${ }^{8}$ Study done at Institute of Dental Sciences and Hospital, Lucknow, India, similar to a study at Taiwan, showed a sensitivity of $91.4 \%$, specificity of $66.6 \%$, positive predictive value of $97.7 \%$, negative predictive value of $33 \%$. The overall diagnostic accuracy of methylene blue stain was $90 \%{ }^{8,9}$

5-ALA uptake is facilitated mainly by decreased ferrochelatase action, enchanced enzymatic activity and reduced intercellular junctions. 5-ALA shows high specificity in comparison to hematoporphyrin derivatives. ${ }^{10}$ Topical 5-ALA can be used on outpatient basis for early detection of malignant lesions as well as fluorescence guided laser resection of oral cancer. The only drawback being the high costs associated in procuring 5-ALA. ${ }^{11}$

Photofrin said to be the most popular sensitizer, is administered exogenously to enhance tumor demarcation by emission of a red fluorescence under wavelength of around $400 \mathrm{~nm} .{ }^{12}$

Topical photofrin used in a study conducted at Chang Gung Memorial Hospital, Taiwan, to identify early oral cancer showed a sensitivity ranging between 92.45 and $93.75 \%{ }^{13}$

In our study, we have used topical 5-ALA stained red fluorescence for demarcating the extent of malignant and premalignant lesions of oral cavity under blue light.

5-aminolevulinic acid hydrochloride can also be used as a form of photodynamic therapy for therapeutic purposes. ${ }^{14}$ Contact endoscopy has been done in laryngeal and pharyngeal malignancies using 5-ALA. ${ }^{15}$ The endoscopy is using narrowband imaging (NBI) and filters in the microscope. ${ }^{15}$ Contact endoscopy in a noninvasive, in vivo microscopic examination done by placing a rigid telescope on mucosa that has been stained by a dye. This produces real time images of the cellular architecture with magnification, thereby eliminating need for biopsywhich is the gold standard for diagnosis. ${ }^{15}$

In a collaborated study involving Taiwan and Japan, comparing white light and narrowband imaging (NBI), detection of carcinoma in situ (CIS) was found to be 
superior in NBI $(\mathrm{p}<0.001) .{ }^{16}$ Study at Italy, utilizing NBI for detection of oral cavity lesions demonstrated a sensitivity and specificity of 96 and $100 \%$ respectively. ${ }^{17}$

Pioneer study of intra-arterial administration of photosensitizer (Photofrin) was conducted at Vilnius University in 1990. This and also the intravenous administration showed a sensitivity of $100 \% .^{12}$

A study done at University of Munich, Germany, utilizing topical ALA solution in the form of gargling, showed a sensitivity of $99 \%$, specificity of $60 \%$, positive predictive value of $77.3 \%$ and a negative predictive value of $97.5 \% .^{11}$

In our study, no spectroscopy was done. As a result, there were no weak positives. Hence, there is a discrepancy with regard to the specificity. Therefore, the sensitivity and specificity in our study was 95.74 and 100\% respectively because biopsy from fluorescent margin (naked eye under blue light) would have been taken only from strongly fluorescent margin.

Study done at Eastman Dental Institute for Oral Healthcare Sciences, London, consisted of 71 patients with clinically suspicious lesions. These patients performed an oral rinse with ALA solution. The sensitivity and specificity for identifying dysplasia or CIS was 83 to $90 \%$ and 79 to $89 \%$ respectively. ${ }^{18}$

In a study done at University of Munich, Germany, topical ALA was used as a rinsing solution. The lesion was examined under 375 to $440 \mathrm{~nm}$ (blue) wavelength of light for fluorescence and biopsy was performed and evaluated for malignancy. Comparable results were observed in white light diagnosis and combined fluorescence diagnosis (CFD) for all biopsy specimens taken. Protoporphyrin IX fluorescence fared best and autofluorescence scored worst for tongue lesions. The best method was said to be a combination of autofluorescence photodetection and topical 5-ALA induced PPIX fluorescence. ${ }^{10}$

Similar to the above study, our study emphasized the efficacy of 5-ALA induced PPIX fluorescence in detection and delineation of tongue malignancies. We observed that all malignancy tongue cases showed $>5 \mathrm{~mm}$ extension beyond visible margin on fluorescence.

In our study, using topical 5-ALA, sensitivity and specificity was found to be 95.74 and 100\% respectively. Positive predictive value was $100 \%$ and negative predictive value was $60 \%$. Diagnostic accuracy was found to be $96 \%$. The data were analyzed using OpenEpi Software Version 2.

To infer, staining with 5-ALA shows autofluorescence and as seen in our study, the lesion may be extending beyond visible margin, and thus a wide excision is mandatory. The average extension in buccal mucosa lesions was $4.35 \mathrm{~mm}(2-7 \mathrm{~mm})$ and in tongue was $11 \mathrm{~mm}(8-13 \mathrm{~mm})$.
The premalignant lesions show an average extension of $3.35 \mathrm{~mm}(2-5 \mathrm{~mm})$. The same autofluorescence using 5-ALA may be used in future to ensure margins of resection during surgery to help bring down the number of local recurrence in oral malignancies.

\section{CONCLUSION}

On staining oral malignant/premalignant lesions with 5-ALA, red fluorescence is observed when seen with blue light (380-450 nm). This ALA induced fluorescence shows the true extent of the lesion in oral malignancies and premalignant lesions. Staining with 5-ALA dye is superior to other supravital dyes like toluidine blue, lugol's iodine and methylene blue in demarcating oral malignant and premalignant lesions.

In our study, topical ALA staining and visualization under blue light showed extension of fluorescence beyond the visible margin in $94 \%$ of patients. The sensitivity and specificity in our study for this fluorescence (extension of lesion beyond visible margin) was 95.74 and $100 \%$. The positive and negative predictive value was 100 and $60 \%$ respectively. Diagnostic accuracy was $96 \%$. The average extension of tumor beyond visible margin was $3.95 \mathrm{~mm}$ in buccal mucosa and $9 \mathrm{~mm}$ in tongue lesions.

In field cancerization, staining with 5-ALA can help to select the appropriate site for biopsy.

5-aminolevulinic acid staining demarcates the malignant and premalignant lesions efficiently and can, therefore, be used while resecting lesions to ensure complete clearance. Further, it also holds promise in photodynamic therapy and laser excision of lesion.

\section{REFERENCES}

1. Petruzzi M, Lucchese A, Baldoni F, Grassi FR, Serpico R. Use of lugol's iodine in oral cancer diagnosis: an overview. Oral Oncol 2010;46(11):811-813.

2. Kumar S, Vezhavendhan N, Reddy S. Assessment of toluidine blue in oral leukoplakia. Int J Clin Dent Sci 2011;2(2):22-24.

3. Niebel $\mathrm{HH}, \mathrm{Chomet} \mathrm{B}$. In vivo staining test for delineation of oral intraepithelial neoplastic change: preliminary report. J Am Dent Assoc 1964;68(6):801-806.

4. Rodriguez CP, Lapiedra CR, Gomez EG, Martinez SL, Warnakulasuriya $S$. The use of toluidine blue in the detection of premalignant and malignant oral lesions. J Oral Pathol Med 2011;40(4):300-304.

5. Warnakulsuriya KA, Johnson NW, et al. Sensitivity and specificity of oral scan toluidine blue rinse in the detection of oral cancer and precancer. J Oral Pathol Med 1996;25(3):97-103.

6. Rosenberg D, Cretin S. Use of meta-analysis to evaluate tolonium chloride in oral cancer screening. Oral Surg Oral Med Oral Pathol 1989;67(5):621-627.

7. Epstein JB, Scully C, Spinelli J. Toluidine blue and lugol's iodine application in the assessment of oral malignant disease and lesions at risk of malignancy. J Oral Pathol Med 1992;21(4): 160-163. 
8. Riaz A, Shreedhar B, Kamboj M, Natarajan S. Methylene blue as an early diagnostic marker for oral precancer and cancer. Springer Plus 2013;2(1):1-7.

9. Chen WY, Lin JS, Cheng HW, Lui MT, Kao SY, Fong Y. Application of in vivo stain of methylene blue as a diagnostic aid in the early detection and screening of oral squamous cell carcinoma and precancer lesions. J Chin Med Assoc 2007; 70(11):497-503.

10. Betz C, Stepp H, Janda P, Arbogast S, Grevers G, Baumgartner $\mathrm{R}$, et al. A comparative study of normal inspection, autofluorescence and 5-ALA induced PPIX fluorescence for oral cancer diagnosis. Int J Cancer 2002;97(2):245-252.

11. Leunig A, Betz C, Mehlmann M,Stepp H, ArbogastS, Grevers G, Baumgartner R. Detection of squamous cell carcinoma of the oral cavity by imaging 5 -aminolevulinic acid induced protoporphyrin IX fluorescence. Laryngoscope 2000;110(1):78-83.

12. Plesniene BL, Mordas A, Cepulis V, Venius J, Navickiene LJ, Rutkovskiene L, et al. Fluorescence diagnostics of oral cancer. Acta Medica Lituanica 2011;18(2):63-69.

13. Chang CJ, Smith WP. Topical application of photofrin for photodynamic diagnosis of oral neoplasms. Plast Reconstr Surg 2005;115(7):1877-1886.
14. Shafirstein G, Friedman A, Siegel E, Moreno M, Baumler W, Yang Fan C, et al. Using 5-Aminolevulinic acid and pulsed dye laser for photodynamic treatment of oral leukoplakia. Arch Otolaryngol Head Neck Surg 2011;137(11):1117-1123.

15. Szeto C, Wehrli B, Whelan F, Franklin J, Nicholas A, Yoo J, et al. Contact endoscopy as a novel technique in the detection and diagnosis of mucosal lesions in the head and neck: a brief review. J Oncol 2011;2011:196302.

16. Lin CY, Watanabe A, Chen CW, Fai Lee K, Lee LI, Wang HW. Narrowband imaging for early detection of malignant tumoursand radiation effect after treatment of head and neck cancer. Arch Otolaryngol Head Neck Surg 2010;136(3): 234-239.

17. Piazza C, Cocco D, Bon FD, Mangili S, Nicolai P, Majorana A, et al. Narrowband imaging and high definition television in evaluation of oral and oropharyngeal squamous cell carcinoma: a prospective study. Oral Oncol 2010;46(4):307-310.

18. Sharwania A, Jerjes W, Salihb V, MacRobert AJ, El-Maaytah M, Khalil HSM, et al. Fluorescence spectroscopy combined with 5-Aminolevulinic acid-induced protoporphyrin IX fluorescence in detecting oral premalignancy. J Photoche Photobiol B 2006;83(1):27-33. 